\title{
Delayed Maturation of the Middle Cerebellar Peduncles at Near-Term Age Predicts Abnormal Neurodevelopment in Preterm Infants
}

\author{
Yong-Ho Choi ${ }^{\mathrm{a}}$ Jong-Min Lee ${ }^{\mathrm{a}}$ Joo Young Lee ${ }^{\mathrm{b}, \mathrm{d}}$ Ji Young Lee ${ }^{\mathrm{c}}$ \\ Young-Jun Lee ${ }^{c}$ Ja Hye Ahn ${ }^{b, d}$ Hyun Ju Lee ${ }^{b, d}$ \\ ${ }^{a}$ Department of Biomedical Engineering, Hanyang University, Seoul, Republic of Korea; ${ }^{b}$ Department of Pediatrics, \\ Hanyang University College of Medicine, Seoul, Republic of Korea; 'Department of Radiology, Hanyang University \\ College of Medicine, Seoul, Republic of Korea; 'Division of Neonatology and Developmental Medicine, Seoul \\ Hanyang University Hospital, Seoul, Republic of Korea
}

\section{Keywords \\ Diffusion tensor imaging • Very-low-birth-weight infant · Cognitive and motor outcomes - Brain microstructure . White matter tracts}

\begin{abstract}
Background: The infant brain grows quickly with elaborate microstructural development during the neonatal period. The white matter, during critical periods of development, is selectively vulnerable to altered maturation and impaired growth in very-low-birth-weight (VLBW) infants. Objective: To evaluate whether abnormal white matter maturation in VLBW infants is associated with poor neurodevelopmental outcomes at 18 months of corrected age. Methods: Between 2015 and 2017, we recruited 60 VLBW infants at 24-32 weeks of gestational age and 15 full-term controls. All participants underwent magnetic resonance imaging at near-term age and were assessed at 18 months of corrected age with the Bayley Scales of Infant and Toddler Development, Third Edition. The associations between regional white matter fractional anisotropy (FA) and mean diffusivity on diffusion tensor imaging (DTI) and developmental outcomes were explored using multivariable linear regression after correcting for gesta-
\end{abstract}

tional age, postmenstrual age at DTI scan, and maternal education level. Results: The FA values of the splenium of the corpus callosum ( $p=0.032)$, corticospinal tract $(p=0.025)$, middle cerebellar peduncle (MCP) $(p<0.001)$, and cingulum $(p=0.043)$ were significantly related to cognitive scores; however, only the association corresponding to the MCP remained significant after correcting for multiple comparisons. The MCP FA $(p=0.008)$ was associated with motor scores after correction for multiple comparisons ( $p=0.008)$. Cognitive impairment (area under the curve $[A \cup C]=0.823$, $95 \%$ confidence interval $[\mathrm{Cl}]=0.722-0.911)$ and motor impairment (AUC $=0.776,95 \% \mathrm{Cl}=0.656-0.899$ ) were predicted by MCP FA. Conclusions: The FA of MCP at near-term age may predict developmental outcomes of VLBW infants at 18 months of corrected age. 02021 The Author(s)

Published by S. Karger AG, Basel

\section{Introduction}

Despite recent improvements in the rates of survival and positive outcomes among preterm children, a substantial proportion of very-low-birth-weight (VLBW; $<1,500 \mathrm{~g}$ ) infants are at high risk for neurodevelopmental
(C) 2021 The Author(s)

Published by S. Karger AG, Basel

This is an Open Access article licensed under the Creative Commons Attribution-NonCommercial-4.0 International License (CC BY-NC) (http://www.karger.com/Services/OpenAccessLicense), applicable to the online version of the article only. Usage and distribution for commercial purposes requires written permission.
Hyun Ju Lee

Department of Pediatrics, Hanyang University Seoul Hospital

222-1 Wangsimni-ro Seongdong-gu

Seoul 04763 (Republic of Korea)

blesslee77@hanmail.net 
impairments in motor, cognition, language, and behavior, even in the absence of significant brain lesions on neuroimaging [1]. Brain magnetic resonance imaging (MRI) scans are usually performed before discharge as a standard of care in VLBW infants and have been considered to provide potential biomarkers of early prognosis [2].

However, the brain regions that are more sensitive to age-specific development and their relative values for predicting the prognoses of VLBW infants via diffusion tensor imaging (DTI) remain controversial. Aside from primary brain injury, total brain volume and secondary dysmaturation of white matter (WM) tracts have been implicated in multiple neurological deficits observed in preterm children. The DTI of early WM structure may provide an earlier and more sensitive biomarker of subsequent aberrant neurodevelopment that complements conventional MRI $[3,4]$. WM fractional anisotropy (FA) in DTI generally increases with brain development and maturation across the neonatal period, reflecting changes in axonal membrane, axonal conduction speeds, and preliminary myelination. Despite evidence of the microstructural alterations causing negative consequences of neurodevelopment in VLBW children, investigations of distinct WM tracts rather than WM microstructure as a whole are lacking. Our group focuses on preterm infants without significant brain injuries to demonstrate these connections with regard to specific WM tracts. Our preliminary study reported that preterm infants who were born with periventricular leukomalacia on conventional MRI and had poor motor outcomes exhibited significantly lower mean FA in the genu of the corpus callosum (gCC) and posterior limb of the internal capsule on DTI scans based on measurements in regions of interest (ROIs) [5]. The probabilistic maps of neonatal functional pathways have been applied to evaluate longitudinal developmental changes in major WM tracts related to preterm birth $[6,7]$. In contrast, little is known about the clinical implications of altered WM pathways associated with later developments of motor deficits, cognitive delay, and limbic systems in preterm neonates with no WM injury or intraventricular hemorrhage. Here, we investigated whether the probabilistic maps of the neonatal WM pathway are affected by preterm birth, which partially explains poor cognitive and motor outcomes in preterm infants. First, we aimed to address the differences in microstructural development in preterm infants compared with healthy full-term infants with normal development. We also assessed whether altered WM tracts in normative preterm infants without significant brain injuries negatively affect developmental outcomes.

\section{Methods}

\section{Subjects}

The present study recruited VLBW infants admitted to Hanyang Seoul Hospital between March 2015 and October 2017 for a prospective preterm cohort study at Hanyang Inclusive Clinic for Developmental Disorders, an affiliate of Hanyang University College of Medicine. We included infants with a birth weight of $<1,500$ $\mathrm{g}$ who underwent scans at near-term age (postmenstrual age [PMA] of 36-41 weeks). The exclusion criteria were as follows: (1) major congenital malformation or chromosomal abnormalities, (2) intrauterine growth retardation, (3) any intraventricular hemorrhage or periventricular leukomalacia as identified with neonatal ultrasound or subsequently performed MRI, (4) necrotizing enterocolitis requiring intestinal resection, or (5) punctate cerebellar hemorrhage. We further enrolled 18 healthy, full-term infants (born between 37 and 42 weeks of gestation) with uneventful deliveries, normal MRI brain scans, and normal neurologic examination results. The following exclusion criteria were employed for full-term infants: chromosomal anomalies, evidence of dysmorphic features, hypoxic-ischemic encephalopathy, congenital infection, and congenital heart disease. The Institutional Review Board (IRB) of Hanyang University Seoul Hospital (IRB No. 2017-04004) approved the study protocol and scanning procedures. Written informed consent was obtained from the parents of all enrolled children.

\section{Neurodevelopmental Assessment}

Neurological and developmental assessments of the children using the Bayley Scales of infant and Toddler Development, Third Edition (BSID-III) [8] were performed at Hanyang Inclusive Clinic for Developmental Disorders at the age of 18 months (corrected for prematurity or at this chronologic age for the full-term infants). The routine examination was performed by a developmental pediatrician, while the developmental assessment was performed by a pediatric physiotherapist, child psychologists, and a neonatologist when the children reached 18 months of age. Developmental outcomes included composite cognitive and motor scores on the BSID-III corrected for the child's adjusted age at the time of evaluation. The normative mean (standard deviation [SD]) for each outcome score was 100. Delayed cognitive and motor development was defined as a cognitive and motor score of $<-1$ SD (i.e., 84 on the BSID-III), respectively.

\section{Magnetic Resonance Imaging}

T1-weighted MRI, T2-weighted MRI, and DTI were performed using a 3.0-T MRI scanner (Philips Real Time Compact Magnet 3.0-Tesla MRI system, Achieva 3.0-Tesla X-series) with a 16-channel SENSE head coil. The MRI scans were obtained at 36-41 weeks of PMA in VLBW infants and 37-40 weeks of PMA in full-term infants during natural sleep and without sedation. Infants were monitored by pulse oximetry during the scan. The T1- and T2weighted images were obtained with sagittal T1 turbo field-echo sequences (TR/TE 8.2/3.8 ms) and axial turbo spin-echo (TR/TE $4,800 / 90 \mathrm{~ms}$ ) sequences, respectively. The DTI sequence parameters were as follows: SENSE factor, $2 ; b$ value, $800 \mathrm{~s} / \mathrm{mm}^{2}$; number of diffusion gradient directions, 15; TR/TE, 8,100/75 ms; 40-50 axial slices with a $2.0-\mathrm{mm}$ thickness; field of view, $224 \mathrm{~mm}$; matrix size, $112 \times 112$; total acquisition time, $6.5 \mathrm{~min}$.
38

Neonatology 2021;118:37-46

DOI: $10.1159 / 000512921$
Choi/Lee/Lee/Lee/Lee/Ahn/Lee 


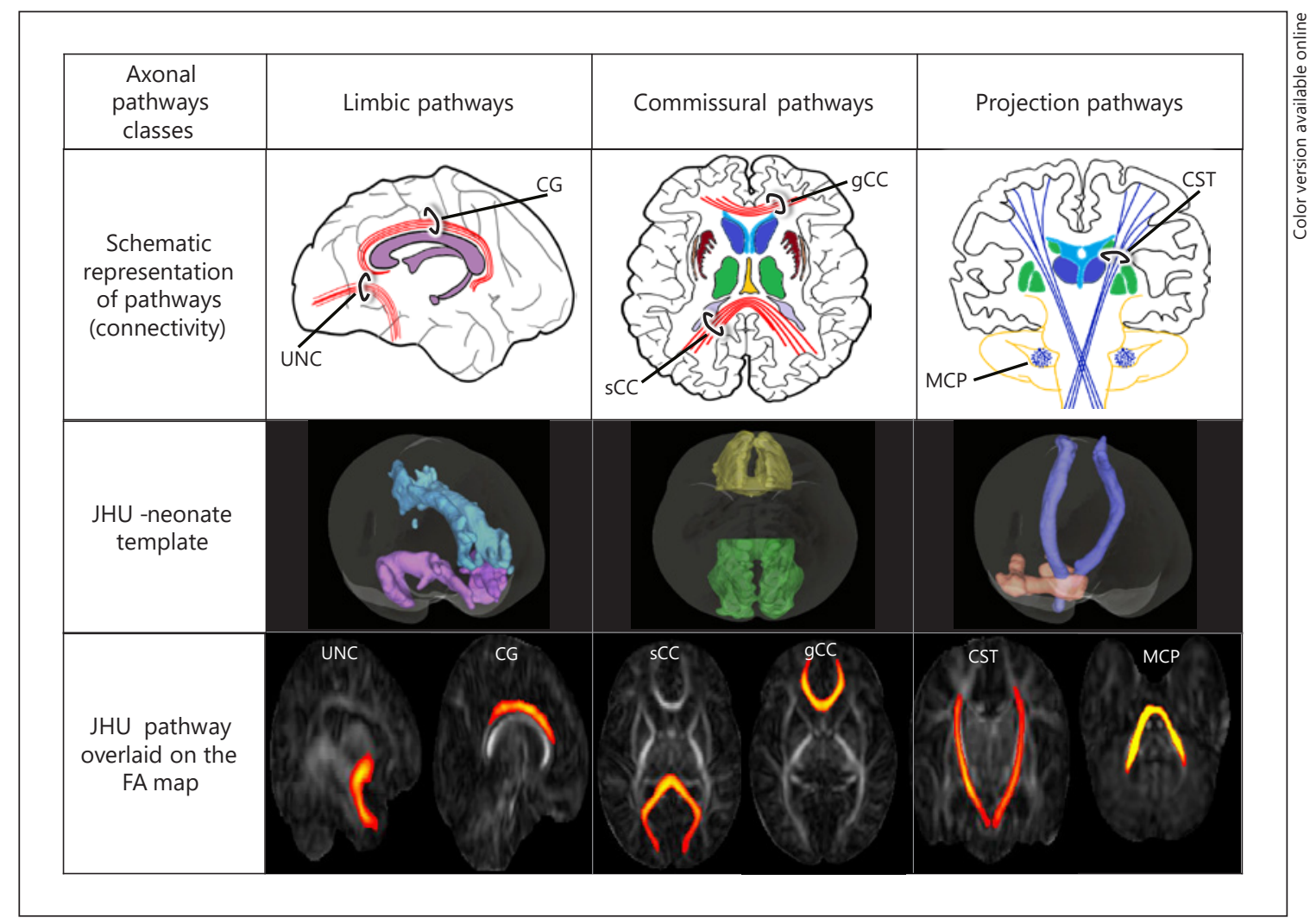

Fig. 1. Probabilistic maps of the white matter tracts comprising the gCC, sCC, CST, MCP, CG, and UNC. JHU, Johns Hopkins University; FA, fractional anisotropy; gCC, genu of corpus callosum; sCC, splenium of corpus callosum; CST, corticospinal tract; MCP, middle cerebellar peduncle; CG, cingulum; UNC, uncinate fasciculus.

\section{Processing of Diffusion MRI Scans}

Diffusion MRI images were processed using FMRIB Software Library (http://www.fmrib.ox.ac.uk/fsl). First, a brain mask was created using the Brain Extraction Tool. Eddy current distortions and motion artifacts were corrected based on the rigid registration using FMRIB's Diffusion Toolbox. A tensor model in each voxel was subsequently reconstructed, and scalar metrics such as FA and mean diffusivity (MD) were calculated. To analyze the microstructure of pathways of interest, we used the probabilistic maps of the fiber pathways obtained from the Johns Hopkins University (JHU) neonate atlas [9]. All FA images were aligned and warped according to the JHU neonate FA atlas using the advanced normalization tools $[10,11]$. We subsequently used established probabilistic maps of fiber pathways constructed with DTI tractography and measured the average trace values of brain regions with a probability of $>10 \%$. The probabilistic maps of $6 \mathrm{WM}$ tracts were overlaid on the JHU neonate atlas to quantify the FA and MD of the pathways of interest related to cognitive, motor, and limbic functions: the gCC and splenium of the corpus callosum (sCC) in commissural pathways, the corticospinal tract (CST) and middle cerebellar peduncle (MCP) in projection pathways for motor function, and the cingulum and uncinate fasciculus in limbic pathways (Fig. 1). The gCC and sCC are the commissural fibers that reflect robust development of interhemi- spheric neocortical connections and are known to mediate cognitive function. The CST and MCP have been linked to motor processes through the motor fibers of the corticospinal tract. The cingulum and uncinate fasciculus are interconnected with the limbic regions of the amygdala and hippocampus involving memory, emotion, and behavior.

\section{Statistical Analysis}

Statistical analyses were performed with SPSS 14.0. To assess clinical characteristics, Fisher's exact and Kruskal-Wallis tests were used to compare proportions and continuous data, respectively. Statistical significance was defined as a $p$ value of $<0.05$. Interhemispheric differences in the CST, MCP, cingulum, and uncinate fasciculus were evaluated with paired $t$ tests. We calculated the mean differences in the FA and MD of the gCC, sCC, CST, MCP, cingulum, and uncinate fasciculus between the preterm and full-term groups from the multiple general linear model after adjusting for PMA at scan. The effect of WM FA and MD on the 18-month neurodevelopmental outcomes was investigated only in preterm infants using regression analysis. A Z-score for BSID-III was used in all regressions in the preterm group. We used a Bonferroni correction of $0.05 / 6=0.008$ for a 2 -sided alpha threshold for the 6 parts of the WM pathways. First, we examined the correlations of FA and MD with 2 outcome scores (the BSID- 
Table 1. Baseline demographic and clinical characteristics of the preterm and term infants included in this study

\begin{tabular}{|c|c|c|c|}
\hline Variables & $\begin{array}{l}\text { Preterm group } \\
(n=60)\end{array}$ & $\begin{array}{l}\text { Term group } \\
(n=15)\end{array}$ & $p$ value \\
\hline GA, weeks & $27.65 \pm 2.58$ & $37.80 \pm 0.77$ & $<0.001$ \\
\hline Birth weight, $g$ & $1,051.75 \pm 269.95$ & $2,995.33 \pm 477.68$ & $<0.001$ \\
\hline Cesarean section & $45(75)$ & $8(53.3)$ & 0.120 \\
\hline Male sex & $26(47.3)$ & $8(57.1)$ & 0.561 \\
\hline SGA & $10(16.3)$ & 0 & 0.196 \\
\hline Age at scanning, weeks & $37.10 \pm 1.71$ & $38.71 \pm 1.10$ & 0.001 \\
\hline BPD moderate/severe & $9(15.8)$ & & \\
\hline Antenatal steroids (full course) & $44(72.1)$ & & \\
\hline Postnatal steroids & $12(20.0)$ & & \\
\hline PDA operated & $21(35.6)$ & & \\
\hline Culture-proven sepsis & $17(28.3)$ & & \\
\hline Age at full enteral feeding, days & $33.55 \pm 28.64$ & & \\
\hline Germinal matrix hemorrhage & $16(26.2)$ & & \\
\hline ROP stage 3 & $8(13.1)$ & & \\
\hline Hearing impairment & $1(1.6)$ & & \\
\hline Maternal age & $33.56 \pm 4.84$ & $33.26 \pm 3.61$ & 0.822 \\
\hline Mother smoking & $1(1.7)$ & 0 & 1.000 \\
\hline \multicolumn{4}{|l|}{ Maternal education } \\
\hline Less than high school & $1(1.7)$ & 0 & 0.413 \\
\hline High school graduate & $12(20)$ & $2(13.3)$ & \\
\hline College/university graduate & $47(78.4)$ & $13(86.6)$ & \\
\hline Age at Bayley III, months & $17.55 \pm 2.64$ & $18.15 \pm 7.23$ & 0.756 \\
\hline Cognitive composite score, mean (SD) & $92.58 \pm 13.78$ & $101.67 \pm 10.47$ & 0.020 \\
\hline Infants with scores $<-1$ SD, $n(\%)$ & $15(25)$ & $0(0)$ & 0.032 \\
\hline Motor composite score, mean (SD) & $92.00 \pm 17.82$ & $108.67 \pm 12.67$ & 0.001 \\
\hline Infants with scores $<-1$ SD, $n(\%)$ & $18(30)$ & $0(0)$ & 0.016 \\
\hline
\end{tabular}

Data are presented as the means \pm SD or $n(\%)$. GA, gestational age; SGA, small gestational age; BPD, bronchopulmonary dysplasia; PDA, patent ductus arteriosus; IVH, intraventricular hemorrhage; MRI, magnetic resonance imaging.

III composite cognitive or motor score) in VLBW infants, adjusting for the PMA at scan in multiple logistic model 1. Gestational age (GA) together at MRI scan was controlled for in the analysis of the effect of WM FA on the developmental outcomes of VLBW infants in multiple logistic model 2. As maternal education level is known to affect neurodevelopmental outcome, it was considered a covariate in multiple logistic model 3. We conducted further analyses to determine if the observed effects might have been further confounded by the environmental factor of maternal education together with the PMA at scan and GA. We defined highrisk infants as VLBW infants who were $>1$ SD below the mean of the BSID-III composite cognitive or motor score. FA was normalized by GA, PMA at DTI scan, and maternal education levels. To ensure the prognostic accuracy for cognitive or motor impairment, a receiver operating characteristic (ROC) curve analysis for the binary classification with logistic regression was performed using sensitivity, specificity, and the area under the curve (AUC) (with 95\% asymptotic normal confidence interval [CI]) of quantitative DTI parameters.

\section{Results}

\section{Infant Characteristics}

Eighty-four participants represented $95 \%$ of eligible infants; 80 of the 84 participants had successful DTI scans. Based on the aforementioned exclusion criteria, 4 infants with intraventricular hemorrhage or periventricular leukomalacia and 3 infants with intrauterine growth retardation were excluded. We enrolled an initial sample of VLBW infants that included 71 datasets. Scans from 6 of the VLBW infants were removed due to poor image quality that precluded DTI processing. Neurodevelopment was assessed with the BSID-III adjusted for prematurity at 18 months of age in 60 of the 65 neonates with DTI scans at near-term age (92\%; 2 children moved and 3 refused to return for the follow-up). Of the 18 full-term infants, 15 were followed up at 18 months of age and completed the BSID-III. Hence, $60 \mathrm{VLBW}$ infants and 15 


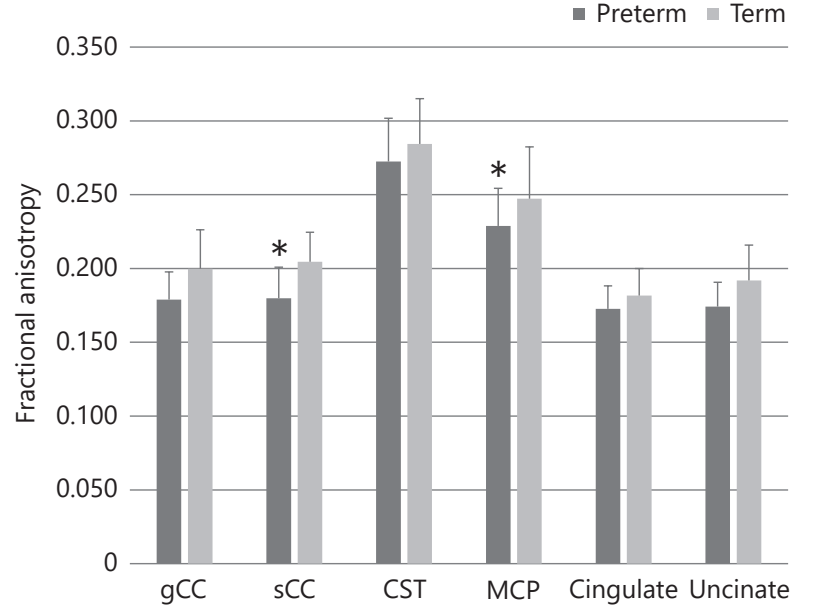

a

$* p<0.008$ vs. term infants, corrected for multiple comparisons

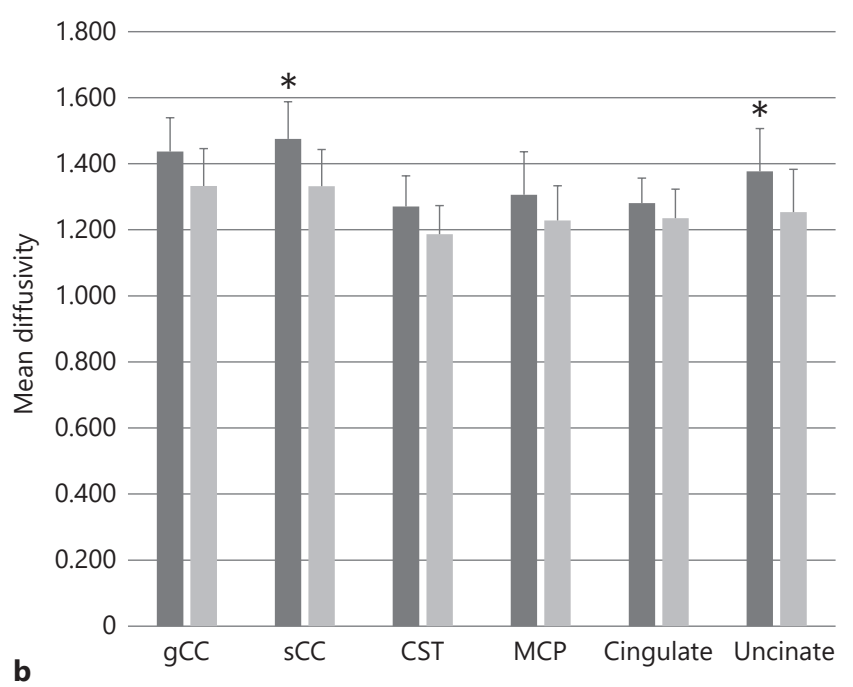

b

Fig. 2. Differences in fractional anisotropy (a) and mean diffusivity (b) between preterm and full-term infants as observed on diffusion tensor imaging. ${ }^{*} p<0.008$ versus full-term infants corrected for multiple comparisons. gCC, genu of corpus callosum; sCC, splenium of corpus callosum; CST, corticospinal tract; MCP, middle cerebellar peduncle.

healthy full-term infants were included in the final cohort. Composite cognitive and motor scores were calculated based on the child's adjusted age at the time of evaluation. Table 1 shows the clinical features of the VLBW infants. The mean GA for the VLBW and full-term infants was $27.6 \pm 2.6$ and $37.8 \pm 0.8$, respectively. Compared with full-term infants, the VLBW infants underwent MRI at an earlier GA (37.7 \pm 1.7 vs. $38.7 \pm 1.1, p=$ 0.001). Male sex, maternal age, and maternal education were comparable between the VLBW and full-term groups. Relative to the full-term controls, the VLBW group exhibited lower cognitive composite score (92.58 \pm 13.78 vs. $101.67 \pm 10.47, p=0.02)$ and motor composite score $(92.00 \pm 17.82$ vs. $108.67 \pm 12.67, p=0.001)$. In the VLBW infants, delayed cognitive (cognitive scores $<-1$ $\mathrm{SD}, 84.5$ ) and motor development (motor scores $<-1 \mathrm{SD}$, $85.5)$ was observed in $15(25 \%)$ and 18 (30\%) infants, respectively.

\section{Differences in FA and MD between VLBW and Full-Term Infants}

Figure 2 shows the differences in FA and MD values of the gCC, sCC, CST, MCP, cingulum, and uncinate fasciculus between the VLBW and full-term infants. As there were no significant differences between the left and right hemispheres of the brain, these values were averaged to yield the mean and interhemispheric FA and MD values. The VLBW infants exhibited lower WM FA compared to full-term infants. The mean FA values of the gCC ( $p=$ $0.002)$, sCC $(p=0.001)$, and $\mathrm{MCP}(p<0.001)$ were significantly decreased, while the mean MD values in the $\operatorname{sCC}(p=0.001)$ and uncinate fasciculus $(p=0.004)$ were increased among VLBW infants relative to full-term infants, after controlling for PMA at MRI scan with a significance level of $p \leq 0.008$ and correcting for multiple comparisons.

\section{Associations between White Matter Development on DTI and Neurodevelopmental Outcomes in VLBW Infants}

In Table 2 , the sCC FA was significantly positively associated with cognitive scores $(p=0.008)$ on the BSID-III at 18 months of corrected age (CA), while the sCC FA was not associated with motor scores $(p=0.010)$ after correcting for PMA at MRI scan in multivariable model 1 (significance level set to $p \leq 0.008$ ). However, the association between the sCC FA and cognitive outcomes remained nonsignificant in multivariable model $2(p=$ $0.024)$ and multivariable model $3(p=0.032)$ after correcting for GA, PMA at MRI scan, and maternal educa- 
Table 2. Multivariate logistic regression analysis of the fiber tracts that showed a significant correlation between FA and each of the 3 Bayley III composite scores (cognitive and motor) at 18 months of corrected age

\begin{tabular}{|c|c|c|c|c|c|c|}
\hline $\begin{array}{l}\text { Fiber tract FA } \\
\text { (independent variables) }\end{array}$ & \multicolumn{3}{|c|}{ Bayley III cognitive score } & \multicolumn{3}{|c|}{ Bayley III motor score } \\
\hline \multicolumn{7}{|l|}{ gCC } \\
\hline Model 1 & 4.165 & $0.710-7.620$ & 0.019 & 3.697 & -1.062 to 8.456 & 0.126 \\
\hline Model 2 & 3.713 & $0.254-7.172$ & 0.036 & 2.648 & -1.945 to 7.241 & 0.254 \\
\hline Model 3 & 3.323 & -0.167 to 6.813 & 0.062 & 2.196 & -2.452 to 6.844 & 0.349 \\
\hline \multicolumn{7}{|l|}{ sCC } \\
\hline Model 2 & 4.246 & $0.584-7.907$ & 0.024 & 4.823 & $0.027-9.619$ & 0.049 \\
\hline Model 3 & 4.003 & $0.357-7.649$ & 0.032 & 4.567 & -0.236 to 9.370 & 0.062 \\
\hline \multicolumn{7}{|l|}{ CST } \\
\hline Model 1 & 4.205 & $0.175-8.235$ & 0.041 & 2.686 & -2.868 to 8.241 & 0.338 \\
\hline Model 2 & 4.799 & $0.854-8.744$ & 0.018 & 3.801 & -1.455 to 9.057 & 0.154 \\
\hline Model 3 & 4.528 & $0.597-8.458$ & 0.025 & 3.501 & -1.761 to 8.763 & 0.189 \\
\hline \multicolumn{7}{|l|}{ Cingulum } \\
\hline Model 1 & 3.845 & $0.620-7.070$ & 0.020 & 3.776 & -0.648 to 8.200 & 0.093 \\
\hline Model 2 & 3.738 & $0.571-6.905$ & 0.021 & 3.553 & -0.633 to 7.740 & 0.095 \\
\hline Model 3 & 3.328 & $0.100-6.556$ & 0.043 & 3.098 & -1.183 to 7.378 & 0.153 \\
\hline \multicolumn{7}{|l|}{ Uncinate fasciculus } \\
\hline Model 1 & 2.867 & -0.498 to 6.232 & 0.094 & 2.610 & -1.982 to 7.201 & 0.261 \\
\hline Model 2 & 2.442 & -0.914 to 5.799 & 0.151 & 1.690 & -2.740 to 6.120 & 0.449 \\
\hline Model 3 & 2.199 & -1.149 to 5.548 & 0.194 & 1.449 & -2.999 to 5.897 & 0.518 \\
\hline
\end{tabular}

Multivariate model 1: adjusted for postmenstrual age at DTI scan; multivariate model 2: adjusted for gestational age and postmenstrual age at DTI scan; multivariate model 3: adjusted for gestational age, postmenstrual age at DTI scan, and maternal education level. Bonferroni-corrected significance level $=0.05 / 6=0.008$. FA, fractional anisotropy; DTI, diffusion tensor imaging; gCC, genu of corpus callosum; sCC, splenium of corpus callosum; CST, corticospinal tract; MCP, middle cerebellar peduncle.

tion level. The gCC FA (cognitive score, $p=0.168$; motor score, $p=0.457$ ), CST (cognitive score, $p=0.041$; motor score, $p=0.338$ ), cingulum (cognitive score, $p=0.020$; motor score, $p=0.093$ ), and uncinate fasciculus (cognitive score, $p=0.094$; motor score, $p=0.261$ ) in multivariable model 1 (significance level set to $p \leq 0.008$ ) were not related to cognitive and motor scores on the BSID-III for infants at 18 months of CA. Furthermore, the associations were not significant after adjusting for GA, PMA at MRI scan, and maternal education level. Only MCP FA remained significant for both cognitive (multivariable models 1, 2, and 3; all $p<0.001$ ) and motor scores (multivariable models 1,2 , and $3 ; p=0.002,0.004$, and 0.008, respectively) in multiple regression analyses with a significance level of $p \leq 0.008$. We found that WM MD had no significant association with either cognitive or motor scores (Table 3.).

\section{ROC Analysis for the Prediction of \\ Neurodevelopmental Outcome with MCP FA in $V L B W$ Infants}

Figure 3 shows the ROC curves corresponding to the use of MCP FA for detecting high-risk VLBW infants. FA was normalized by GA, PMA at DTI scan, and maternal education levels. High-risk VLBW infants were defined according to cognitive or motor scores below $1 \mathrm{SD}$ of the mean in BSID-III at 18 months. The ROC analysis of MCP FA for predicting cognitive impairment adjusted for PMA at DTI scan yielded an AUC of $0.722(95 \% \mathrm{CI}=$ 0.617-0.846). After adjusting for GA, PMA at DTI scan, and maternal education levels, the ROC analysis of MCP FA identified diagnostic accuracy with maximum discrimination (AUC $=0.823,95 \% \mathrm{CI}=0.722-0.911$ ), thus predicting cognitive impairment. The ROC curves of MCP FA for predicting motor impairments yielded an 
Table 3. Multivariate logistic regression analysis of each fiber tract showing a significant correlation between MD and each of the 3 Bayley III composite scores (cognitive and motor) at 18 months of corrected age

\begin{tabular}{|c|c|c|c|c|c|c|}
\hline $\begin{array}{l}\text { Fiber tract MD } \\
\text { (independent variables) }\end{array}$ & \multicolumn{3}{|c|}{ Bayley III cognitive score } & \multicolumn{3}{|c|}{ Bayley III motor score } \\
\hline Model 1 & -2.653 & -6.453 to 1.147 & 0.168 & -1.940 & -7.107 to 3.228 & 0.457 \\
\hline Model 2 & -2.494 & -6.230 to 1.242 & 0.187 & -1.608 & -6.505 to 3.289 & 0.515 \\
\hline Model 3 & -2.244 & -5.959 to 1.471 & 0.232 & -1.334 & -6.229 to 3.560 & 0.588 \\
\hline Model 2 & -2.688 & -6.523 to 1.146 & 0.166 & -1.561 & -6.595 to 3.472 & 0.538 \\
\hline Model 3 & -2.431 & -6.244 to 1.383 & 0.208 & -1.277 & -6.309 to 3.754 & 0.614 \\
\hline \multicolumn{7}{|l|}{ CST } \\
\hline Model 1 & -2.306 & -6.170 to 1.558 & 0.238 & -1.074 & -6.324 to 4.175 & 0.684 \\
\hline Model 2 & -2.404 & -6.191 to 1.383 & 0.210 & -1.272 & -6.236 to 3.691 & 0.611 \\
\hline Model 3 & -2.423 & -6.164 to 1.319 & 0.201 & -1.292 & -6.229 to 3.645 & 0.603 \\
\hline \multicolumn{7}{|l|}{ Cingulum } \\
\hline Model 1 & -1.342 & -5.050 to 2.367 & 0.473 & 0.082 & -4.935 to 5.095 & 0.974 \\
\hline Model 2 & -1.861 & -5.521 to 1.799 & 0.314 & -0.927 & -5.709 to 3.854 & 0.700 \\
\hline Model 3 & -1.517 & -5.171 to 2.137 & 0.410 & -0.546 & -5.342 to 4.250 & 0.821 \\
\hline \multicolumn{7}{|l|}{ Uncinate fasciculus } \\
\hline Model 1 & -2.810 & -6.614 to 0.994 & 0.145 & -2.393 & -7.573 to 2.787 & 0.360 \\
\hline Model 2 & -2.182 & -6.010 to 1.645 & 0.259 & -1.008 & -6.047 to 4.030 & 0.691 \\
\hline Model 3 & -1.991 & -5.795 to 1.812 & 0.300 & -0.819 & -5.861 to 4.222 & 0.747 \\
\hline
\end{tabular}

Multivariate model 1: adjusted for postmenstrual age at DTI scan; multivariate model 2: adjusted for gestational age and postmenstrual age at DTI scan; multivariate model 3: adjusted for gestational age, postmenstrual age at DTI scan, and maternal education level. Bonferroni-corrected significance level $=0.05 / 6=0.008$. MD, mean diffusivity; DTI, diffusion tensor imaging; gCC, genu of corpus callosum; sCC, splenium of corpus callosum; CST, corticospinal tract; MCP, middle cerebellar peduncle.

AUC of 0.646 (95\% CI $=0.495-0.809)$ after adjusting for PMA at DTI scan. After adjustments for GA, PMA at DTI scan, and maternal education levels, MCP FA significantly helped to predict motor impairment with a higher accuracy than MCP FA after adjusting for PMA at DTI scan (AUC $=0.776,95 \% \mathrm{CI}=0.656-0.899)$.

\section{Discussion}

The present investigation evaluated the relationship between DTI at near-term age and later neurodevelopmental outcomes in VLBW children. MRI indicated that none of the enrolled VLBW infants featured any apparent brain abnormalities; however, the low sCC and MCP FA and high sCC and uncinate $\mathrm{MD}$ of these infants relative to their full-term counterparts suggest that the former ex- hibited impaired brain development. Furthermore, the FA values of the MCP in VLBW infants showed a positive association with cognition and motor performance at 18 months of CA when adjusted for GA, PMA at DTI scan, and maternal education levels.

The rapidly growing neonatal brain is not a homogenous organ: regions and processes feature different developmental trajectories. Prior reports have indicated that the WM develops in ventral-to-dorsal and central-to-peripheral directions, thereby suggesting that vulnerabilities in a given region may be related to local disruptions and allowing for more accurate predictions of risk to later neurodevelopment $[7,12]$. An atlas-based DTI analysis of 66 VLBW infants conducted by Rose et al. [13] demonstrated that the microstructure of the posterior limb of the internal capsule (PLIC), which undergoes myelination at near-term age, may feature the strongest prognos- 


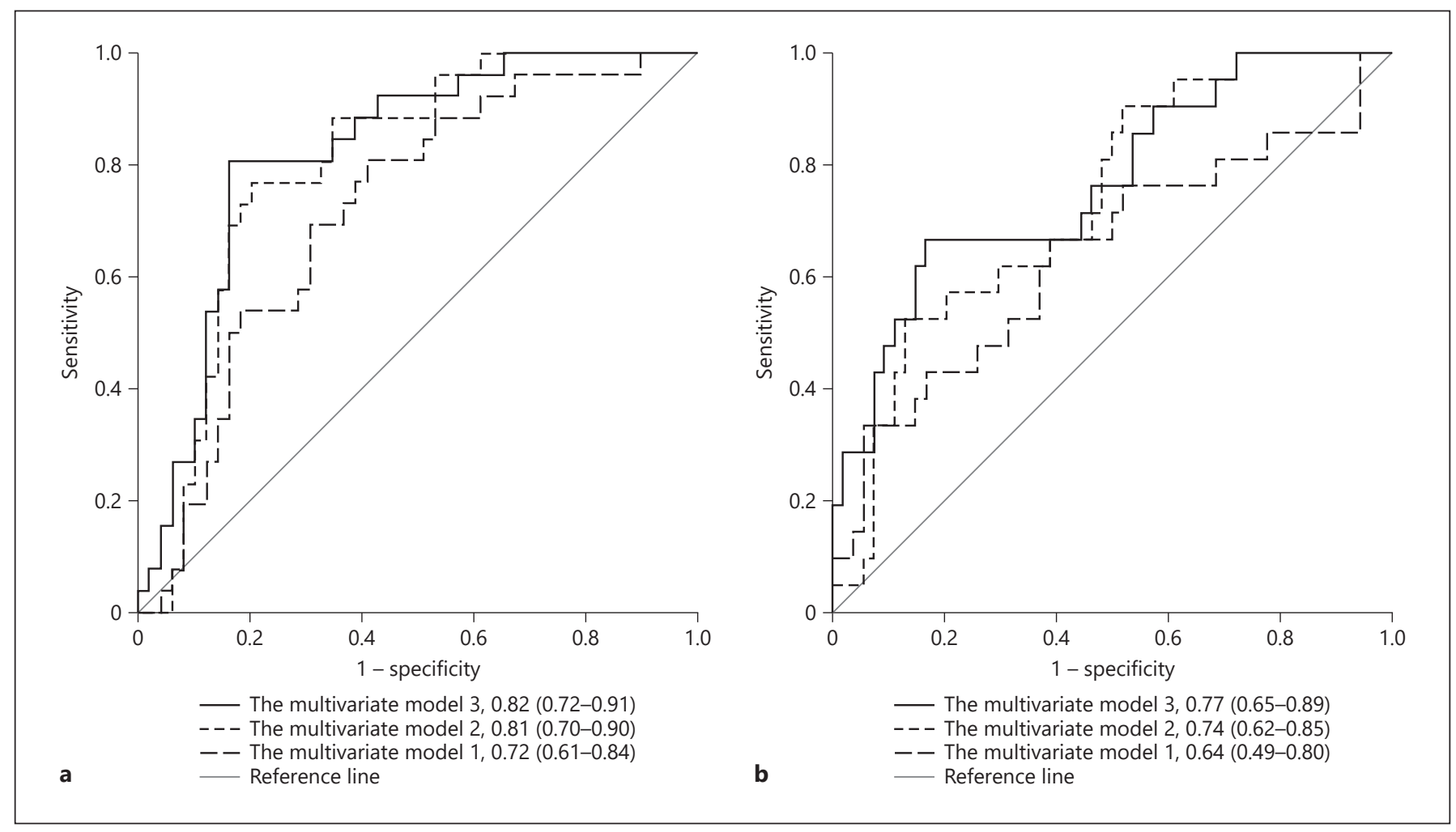

Fig. 3. Receiver operator characteristic curves for the prediction of BSID-III cognitive (a) and motor (b) composite scores below 1 standard deviation of the mean in very-low-birth-weight preterm children at 18 months of corrected age using logistic regression based on near-term middle cerebellar peduncle fractional anisot-

tic value for future neurodevelopment. In the present study, the CST in the probabilistic map of WM pathways has not been linked to aberrant motor or cognitive impairment despite its documented mediation of motor processes through the motor fibers of the PLIC. Our inclusion of a cohort of normative VLBW neonates without brain injury may explain this discrepancy. As 47 of the 102 preterm neonates included in the investigation by Rose et al. [13] exhibited WM injury and cerebellar abnormalities identified on conventional structural MRI, we surmise that significant WM abnormalities dominate the PLIC microstructure of representative motor tracts more when compared to those of children with normal WM. The corpus callosum (CC) and cerebellum have been previously demonstrated to have abnormal growth trajectories relative to controls in VLBW children [14, 15]. CC WM microstructure at term-equivalent age was previously reported to correlate with cognitive function at 2 years of adjusted age [14]. Cahill-Rowley et al. [15] reported that gCC assessed with DTI contributed to best ropy on DTI. Multivariable model 1, adjusted for postmenstrual age at DTI scan; multivariable model 2 , adjusted for gestational age and postmenstrual age at DTI scan; multivariable model 3, adjusted for gestational age, postmenstrual age at DTI scan, and maternal education level. DTI, diffusion tensor imaging.

prediction for gait impairment in VLBW infants at 18-22 months of adjusted age. Cerebellar abnormalities on conventional MRI were associated with early neurodevelopment and gait, reflecting an underrecognized role in cerebellar contributions to cognition and motor function [13].

As several MRI techniques have explored the organization and maturation of cerebellar WM in the fetal and infant brain, cerebellar injury is increasingly recognized as a complication of premature birth. Impaired cerebellar development in the absence of direct cerebellar injury has been reported following extreme preterm birth [16]. Our previous volumetric MRI analyses of the differences in cerebellar volume between VLBW and full-term infants revealed the importance of neonatal cerebellar growth and WM microstructure in VLBW infants [17]. The dynamic cerebellar growth of 5 -fold in volume and 30 -fold in surface area between 24 and 40 weeks of GA may be attributed to an increased vulnerability of the cerebellum to early developmental insults during late gestation. Sev- 
eral long-term studies of preterm infants extending from early childhood into adulthood have shown a relationship between cerebellar volume and neurodevelopment [18]. The present assessment of the microstructural properties of the MCP with diffusion MRI at a full-term equivalent age emphasizes the importance of cerebellar underdevelopment in a normative dataset of VLBW neonates with no WM injury or intraventricular hemorrhage. Notably, the MCP seems to be an important developmental link between the cerebrum and the cerebellum [19]. The impairment of cerebellar peduncles in VLBW infants has also been linked to direct cerebellar injury and remote effects of alteration in the cerebello-cerebral circuit $[16,20]$.

The VLBW infants exhibited developmental alterations in several brain regions. In the present study, the relationship between sCC FA and cognitive scores was significant when correcting for age at MRI scan, but no longer significant after controlling for prematurity as well as age at MRI scan. This result shows that the amount of prematurity in even VLBW group is related to reduced FA, which potentially affects cognitive scores. Although impaired neurodevelopment in VLBW infants is commonly related to periventricular leukomalacia and intraventricular hemorrhage, evidence suggests that alterations of the CC observed on MRI, even in the absence of major focal lesions, are key to the identification of motor and cognitive impairment in children born preterm [18, 21 . The volume and FA of the CC have been found to be affected by prematurity, which partially explains the poor cognitive and motor outcomes [22, 23].

While maps of WM tracts are a valuable tool to examine developmental alterations of each WM pathway, the clinical significance of the cerebellum tracts has not been correlated with subsequent neurobehavioral and cognitive outcomes in follow-up studies [13]. However, the present study has several limitations. The small sample size with no sample size calculation and the relatively wide range at time of MRI scan may lead to subject heterogeneity and skew interpretation of the data. Second, the follow-up at 18 months of CA is still quite young for neurodevelopmental assessment, where dynamic clinical development can potentially change across age and should have been considered in the analysis of this study. Sequential neuroimaging at term-equivalent age and 4 months of adjusted GA will contribute to a more accurate prediction of adverse developmental outcomes.

DTI in VLBW infants would benefit from investigations of microstructural development in distinct WM tracts to predict neurodevelopmental outcomes. A strength of our DTI study includes the exploration of al- tered cerebellar WM tracts and the focus on infants without significant brain injuries. If cerebellar development at near-term age could be determined early as prognostic biomarkers, targeted interventions could potentially promote earlier access to efficient treatment and prevent neurodevelopmental disabilities to improve quality of life in children born with VLBW.

\section{Statement of Ethics}

The Institutional Review Board (IRB) of Hanyang University Seoul Hospital (IRB No. 2017-04-004) approved the study proto$\mathrm{col}$ and scanning procedures. Written informed consent was obtained from the parents of all the enrolled children.

\section{Conflict of Interest Statement}

None of the authors has conflicts of interest to disclose.

\section{Funding Sources}

This work was supported by the National Research Foundation of Korea Grant funded by the Korean Government MSIT (NRF2020-R1F1A1048529 and M3E5D9080787) and the research fund of Hanyang University (HY-2018).

\section{Author Contributions}

H.J.L. drafted the manuscript and made substantial contributions to the design of this study. Y.H.C., J.M.L., Y.J.L., and J.Y.L. participated in data acquisition and performed data analysis. J.Y.L. and J.H.A. were active in research conception and critical revision of the manuscript.

References

Neonatology 2021;118:37-46

1 Peralta-Carcelen M, Carlo WA, Pappas A, Vaucher YE, Yeates KO, Phillips VA, et al. Behavioral problems and socioemotional competence at 18 to 22 months of extremely premature children. Pediatrics. 2017;139(6): e20161043.

2 Keunen K, Išgum I, van Kooij BJ, Anbeek P, van Haastert IC, Koopman-Esseboom C, et al. Brain volumes at term-equivalent age in preterm infants: imaging biomarkers for neurodevelopmental outcome through early school age. J Pediatr. 2016;172:88-95.

3 Schadl K, Vassar R, Cahill-Rowley K, Yeom $\mathrm{KW}$, Stevenson DK, Rose J. Prediction of cognitive and motor development in preterm children using exhaustive feature selection and cross-validation of near-term white matter microstructure. Neuroimage Clin. 2018; 17:667-79. 
4 Huppi PS, Maier SE, Peled S, Zientara GP, Barnes PD, Jolesz FA, et al. Microstructural development of human newborn cerebral white matter assessed in vivo by diffusion tensor magnetic resonance imaging. Pediatr Res. 1998;44(4):584-90.

5 Kim DY, Park HK, Kim NS, Hwang SJ, Lee HJ. Neonatal diffusion tensor brain imaging predicts later motor outcome in preterm neonates with white matter abnormalities. Ital J Pediatr. 2016;42(1):104.

6 Akazawa K, Chang L, Yamakawa R, Hayama S, Buchthal S, Alicata D, et al. Probabilistic maps of the white matter tracts with known associated functions on the neonatal brain atlas: application to evaluate longitudinal developmental trajectories in term-born and preterm-born infants. Neuroimage. 2016;128: 167-79.

7 Rose J, Vassar R, Cahill-Rowley K, Guzman XS, Stevenson DK, Barnea-Goraly N. Brain microstructural development at near-term age in very-low-birth-weight preterm infants: an atlas-based diffusion imaging study. Neuroimage. 2014;86:244-56.

8 Bayley N, Reuner G. Bayley scales of infant and toddler development: Bayley-III. Frankfurt am Main: Pearson; 2014.

9 Oishi K, Mori S, Donohue PK, Ernst T, Anderson L, Buchthal S, et al. Multi-contrast human neonatal brain atlas: application to normal neonate development analysis. Neuroimage. 2011;56(1):8-20.

10 Avants BB, Epstein CL, Grossman M, Gee JC. Symmetric diffeomorphic image registration with cross-correlation: evaluating automated labeling of elderly and neurodegenerative brain. Med Image Anal. 2008;12(1):26-41.
11 Avants BB, Tustison NJ, Song G, Cook PA, Klein A, Gee JC. A reproducible evaluation of ANTs similarity metric performance in brain image registration. Neuroimage. 2011;54(3): 2033-44.

12 Dubois J, Hertz-Pannier L, Dehaene-Lambertz G, Cointepas Y, Le Bihan D. Assessment of the early organization and maturation of infants' cerebral white matter fiber bundles: a feasibility study using quantitative diffusion tensor imaging and tractography. Neuroimage. 2006;30(4):1121-32.

13 Rose J, Cahill-Rowley K, Vassar R, Yeom KW, Stecher X, Stevenson DK, et al. Neonatal brain microstructure correlates of neurodevelopment and gait in preterm children $18-22 \mathrm{mo}$ of age: an MRI and DTI study. Pediatr Res. 2015;78(6):700-8.

14 van Kooij BJ, de Vries LS, Ball G, van Haastert IC, Benders MJ, Groenendaal F, et al. Neonatal tract-based spatial statistics findings and outcome in preterm infants. AJNR Am J Neuroradiol. 2012;33(1):188-94

15 Cahill-Rowley K, Schadl K, Vassar R, Yeom KW, Stevenson DK, Rose J. Prediction of gait impairment in toddlers born preterm from near-term brain microstructure assessed with DTI, using exhaustive feature selection and cross-validation. Front Hum Neurosci. 2019; 13:305.

16 Volpe JJ. Cerebellum of the premature infant: rapidly developing, vulnerable, clinically important. J Child Neurol. 2009;24(9):1085104.
17 Lee JM, Choi YH, Hong J, Kim NY, Kim EB, Lim JS, et al. Bronchopulmonary dysplasia is associated with altered brain volumes and white matter microstructure in preterm infants. Neonatology. 2019;116(2):163-70.

18 Duerden EG, Foong J, Chau V, Branson H, Poskitt KJ, Grunau RE, et al. Tract-based spatial statistics in preterm-born neonates predicts cognitive and motor outcomes at 18 months. AJNR Am J Neuroradiol. 2015;36(8): 1565-71.

19 Travis KE, Leitner Y, Feldman HM, BenShachar M. Cerebellar white matter pathways are associated with reading skills in children and adolescents. Hum Brain Mapp. 2015; 36(4):1536-53.

20 Limperopoulos C, Chilingaryan G, Sullivan N, Guizard N, Robertson RL, du Plessis AJ. Injury to the premature cerebellum: outcome is related to remote cortical development. Cereb Cortex. 2014;24(3):728-36.

21 Nosarti C, Rushe TM, Woodruff PW, Stewart AL, Rifkin L, Murray RM. Corpus callosum size and very preterm birth: relationship to neuropsychological outcome. Brain. 2004; 127(Pt 9):2080-9.

22 Thompson DK, Inder TE, Faggian N, Warfield SK, Anderson PJ, Doyle LW, et al. Corpus callosum alterations in very preterm infants: perinatal correlates and 2 year neurodevelopmental outcomes. Neuroimage. 2012;59(4):3571-81.

23 Malavolti AM, Chau V, Brown-Lum M, Poskitt KJ, Brant R, Synnes A, et al. Association between corpus callosum development on magnetic resonance imaging and diffusion tensor imaging, and neurodevelopmental outcome in neonates born very preterm. Dev Med Child Neurol. 2017;59(4):433-40. 